\title{
STEEL FIBER CURVATURE IN CONCRETE COMPOSITES: MODULUS PREDICTIONS USING EFFECTIVE STEEL FIBER PROPERTIES
}

\author{
Abdellatif Selmi \\ Department of Civil Engineering, Ecole Nationale d’Ingénieurs de Tunis, B.P.37, Le belvédère 1002, Tunis, Tunisia
}

Received 2013-09-20; Revised 2013-10-01; Accepted 2013-12-13

\begin{abstract}
Results in the literature demonstrate that substantial improvements in the mechanical behavior of concrete have been attained through the addition of steel fibers as a reinforcing phase. We have developed a model combining finite element results and micromechanical methods to determine the effective reinforcing modu-lus of hook-ended steel fibers. This effective reinforcing modulus is then used within a multiphase micro-mechanics model to predict the effective modulus of concrete reinforced with a distribution of fibers. We found that fiber curvature effect is negligible when compared to straight fibers. Then mechanical properties of concrete reinforced with crimped steel fibers are predicted using Weng and Huang schemes. The predic-tions are in excellent agreement with experimental results.
\end{abstract}

Keywords: Steel Fiber, Concrete, Mechanical Properties, Modeling, FE

\section{INTRODUCTION}

Highlight Plain concrete possesses a very low tensile strength, limited ductility and little resistance to cracking (Naaman, 2003; Dasari et al., 2012). Internal micro cracks are inherently present in the concrete and its poor tensile strength is due to the propagation of such micro cracks, eventually leading to brittle fracture of the concrete (Dasari et al., 2012). It has been recognized that when reinforced with small, closely spaced and uniformly distributed fibers, gets strengthened enormously, thereby rendering the matrix to behave as a composite material with properties significantly different from conventional concrete (Naaman, 2003; Pawade et al., 2011a; Pant and Parekar, 2009).

The addition of fibers to the concrete would provide a better control of the crack initiation and its subsequent growth and propagation to improve the structural durability and would substantially increase elastic modulus and decrease brittleness (Naaman, 2003; Pawade et al., 2011b; Pant and Parekar, 2009). Moreover, fiber reinforcement enhances the impact and fatigue resistance of concrete structures (Luca et al., 2006).
Because of the vast improvements achieved by the addition of fibers to concrete, there are several applications where Fiber Reinforced Concrete (FRC) can be intelligently and beneficially used (Oslejs, 2008). Steel fibers are particularly suitable for structures when they are subjected to loads over the serviceability limit state in bending and shear and when exposed to impact or dynamic forces, as they occur under seismic or cyclic action (Pawade et al., 2011a; Neves and Fernandes, 2005). These fibers have already been used in many large projects involving the construction of industrial floors, pavements, highwayoverlays, parking areas, airport runways, floors resting on soil, floor slabs, walls and foundations (Luca et al., 2006; Pant and Parekar, 2009). To help obtaining uniform fiber dispersion in the matrix and improve strength and the bonding between fiber and matrix Silica fume can be introduced (Pawade et al., 2011b).

Toughness of steel fiber reinforced silica fume concrete under compression and dynamic action was done by Ramadoss et al. (2009). They quantify the effect of fiber on compressive strength of concrete in terms of fiber reinforcing parameter.

Fiber characteristics such as their type, shape, volume content $\mathrm{V}_{\mathrm{f}}$ and aspect ratio $\mathrm{L} / \mathrm{D}$, where $\mathrm{L}$ is the fiber 
length and $\mathrm{D}$ is the fiber diameter play an important role in modifying the behavior of concrete (Pawade et al., 2011b). Steel fibers are available in the varied shapes and sizes (Naaman, 2003). They are also available in the form of glue boards, which facilitates the introduction into the concrete and prevents the formation of sea urchin. The steel fiber aspect ratio is often between 30 and 150 for a length ranging from 6.4 to $76 \mathrm{~mm}$.

Many experimental and theoretical researches have been done in order to quantify the performance enhancement of concrete when reinforced with steel fibers. However, there is still incomplete knowledge on the design/ analysis of FRC structural members.

In this study, we develop finite elementmicromechanical model. First, direct Finite Element (FE) based numerical approach and Voigt homogenization method are used to determine the effective reinforcing modulus $\left(\mathrm{E}_{\mathrm{ERM}}\right)$ of an embedded hook-ended fiber which is a representative value denoted that accounts for the reduction in reinforcement provided by the curved fiber in comparison to the reinforcement provided by a straight fiber.

Second, using the hook-ended steel fiber effective reinforcing modulus, steel fiber concrete composite mechanical properties are predicted using two mean field homogenization models; Weng and Huang models. Then the predicted data are compared to experimental results and the steel fiber curvature effect is deduced.

Third, the mechanical properties of crimped steel fiber reinforced concrete composites for different aspect ratio and at different concrete ages are delivered using Weng and Huang models.

This is the main contribution of the study. Indeed, while most papers using analytical methods to predict the reinforcing effect of steel fiber on concrete consider that fibers are straight, in the present work we take the fiber curvature into account using finite elementmicromechanical model. This approach will highlight the impact of fiber curvature on the effective modulus of a FRC. Once the fiber effective modulus is predicted, two homogenization schemes are used in order to predict various shape fiber concrete composite properties. These are compared to experimental results.

The study has the following outline. In section 2 , the finite element method is exposed, the micromechanical schemes are summarized and numerical predictions of hook-ended fiber concrete composite mechanical properties are delivered. In section 3, several numerical predictions of crimped fiber concrete composite mechanical properties are presented. Conclusions are discussed in section 4 .

\section{FINITE ELEMENT AND MICROMECHANICAL SCHEMES}

The issues which will be clarified above indicate several key aspects that will influence the ability to design and model materials that fully exploit steel fibers. One issue which has not been taken in consideration in the modeling of FRCs, but which seems critical, is the characteristic curvature of fibers. To address this question, we have developed a model that integrates the curvature of the fibers into micromechanical predictions of the FRC effective modulus; hook-ended steel fiber is the illustrative example (Fig. 1). Hence, in this section we will focus on the use of hook-ended steel fibers as the reinforcing phase in a bulk concrete material (Neves and Fernandes, 2005).

The properties of 3D randomly oriented hookended fibers are: Length $\mathrm{L}=30 \mathrm{~mm}$, diameter $\mathrm{D}=$ $0.38 \mathrm{~mm}$, Poisson's ratio: $\mathrm{V}_{\mathrm{f}}=0.3$, Elastic modulus: $\mathrm{E}_{\mathrm{f}}=210000 \mathrm{MPa}$.

Properties of silica fume concrete at 42 days of age: Poisson's ratio: $\mathrm{v}_{\mathrm{c}}=0.2$. Young's modulus: $\mathrm{E}_{\mathrm{c}}=35800$ MPa (Neves and Fernandes, 2005).

\subsection{Finite Element-Micromechanical Model Presentation}

Based on the previous discussion, we are interested in using micromechanical techniques to study the effective elastic modulus of fiber-reinforced concrete. The basis of the model is to determine the $\mathrm{E}_{\mathrm{ERM}}$ of an embedded curved fiber. Thus while the fiber modulus is a material property, the $\mathrm{E}_{\mathrm{ERM}}$ is a material parameter that is a function of the geometry of the fiber curvature. This effective modulus is then available for use in standard micromechanical models in lieu of the true (actual) fiber modulus. The individual phase materials are modeled as linear elastic and is otropic and perfect bonding between the phases is assumed. The details of the finite element analysis of an embedded curved fiber follow. Next, the formulation of the MoriTanaka and consequently Weng and Huang methods for a multiphase composite with randomly oriented straight fibers in three-dimensional space will be presented.

\subsection{Finite Element Analysis}

To determine the $\mathrm{E}_{\mathrm{ERM}}$ of an embedded steel fiber, ANSYS program was used to create and analyze a threedimensional finite element model of a single, infinitely long steel fiber perfectly bonded within a concrete material. For axial loading, the problem is symmetric about the $\mathrm{x}=$ 0 and $\mathrm{z}=0$ planes; thus the quarter-symmetric unit cell shown in Fig. 2 and 3 is used for the analysis. 


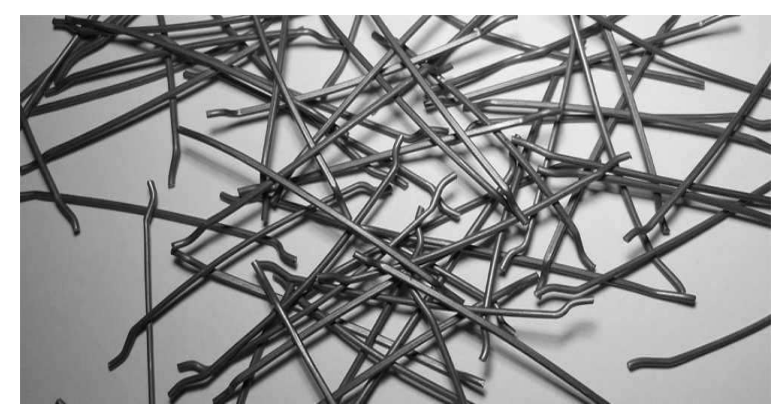

Fig. 1. Schematic representation of hook-ended steel fibers

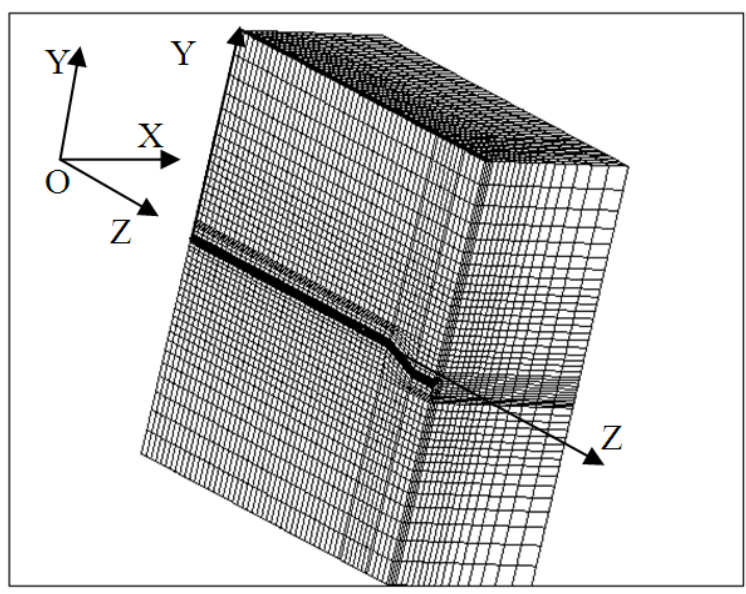

Fig. 2. 3D view of FE mesh corresponding to $0.0015 \%$ of steel fiber

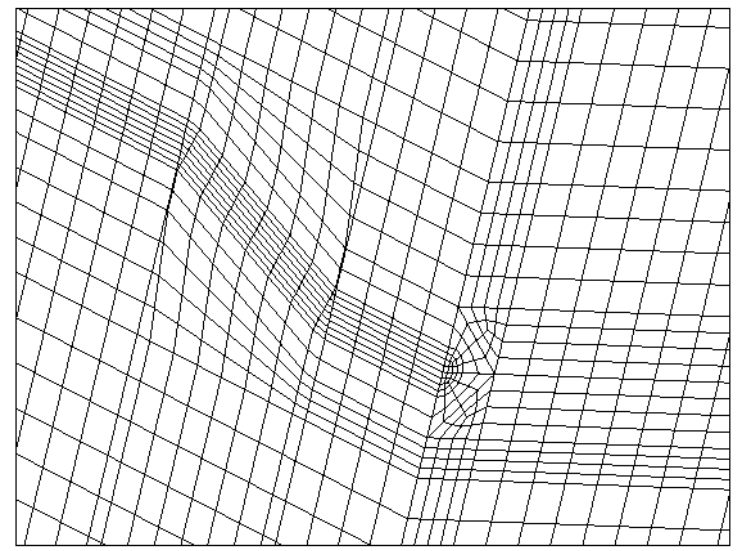

Fig. 3. Zoom of 3D view of $\mathrm{FE}$ mesh corresponding to $0.0015 \%$ of steel fiber showing the steel fiber curvature

The size of the cell was chosen such that the effective cell response is independent of additional matrix material. The models used for actual analysis have volume fractions on the order of $0.0015 \%$ to ensure that the matrix appears infinitely large to the steel fiber. The model is built from a series of 4 sided areas. The volumes of the model are then constructed by connecting these area sets with a series of lines and arcs; arcs are used for the fiber volumes to reflect the curved surfaces while straight lines are used for the remainder. The volumes are meshed using 20 node bricks containing midside nodes. This allows element edges to be parabolic in shape, which accurately captures the curved surfaces.

For the finite element simulations symmetry conditions $\mathrm{u}_{\mathrm{x}}=0$ and $\mathrm{u}_{\mathrm{x}}=0$ were prescribed on the $x=0$ and $z=0$ planes, respectively. The model was constrained in the y direction at a single point to prevent free body translation and an infinitesimally small axial displacement, $\Delta$, prescribed to all nodes on the plane $\mathrm{z}=\frac{1}{2}$ The effective modulus of the cell is defined as Equation (1):

$\mathrm{E}_{\mathrm{cell}}^{\mathrm{FE}}=\frac{\mathrm{F}_{\text {tot }} \mathrm{L}}{2 \mathrm{~A} \Delta}$

$F_{\text {tot }}$ is the sum of all nodal resultant forces on the displaced plane and $\mathrm{A}$ is the cross-sectional area of the cell.

In the present work, $\frac{F_{\text {tot }}}{\Delta}=90486941214 \mathrm{~N} / \mathrm{mm}$ which result in $\mathrm{E}_{\text {cell }}^{\mathrm{FE}}=35802612805 \mathrm{MPa}$.

In order to extract the $\mathrm{E}_{\mathrm{ERM}}$ of the embedded curved fiber (as it exists in the matrix) from (1), we use the Voigt model for the effective cell response Equation (2):

$\mathrm{E}_{\text {cell }}^{\text {parallel }}=\mathrm{V}_{\mathrm{f}} \mathrm{E}_{\mathrm{ERM}}+\left(1-\mathrm{V}_{\mathrm{f}}\right) \mathrm{E}_{\mathrm{c}}=\mathrm{E}_{\text {cell }}^{\mathrm{FE}}$

Where:

$V_{f}=$ The fiber volume fraction within the finite element cell

$\mathrm{E}_{\mathrm{c}}=$ The concrete modulus

From (1) and (2), $\mathrm{E}_{\mathrm{ERM}}$ of the embedded steel fiber can be calculated as:

$E_{E R M}=\frac{E_{\text {cell }}^{\mathrm{FE}}-\left(1-V_{f}\right) E_{c}}{V_{f}}=209987 \mathrm{MPa}$

\subsection{Interpretation}

$\mathrm{E}_{\mathrm{ERM}}$ can be considered to represent the modulus of a straight fiber that, under identical loading conditions, would yield the same effective cell response as that 
obtained with the reel steel fiber. The benefit of such an analysis is that it allows the hook-ended fiber curvature to be integrated into traditional micromechanics techniques by simply modeling the fiber as straight inclusions with a reduced reinforcing modulus based on the embedded geometry and determined via finite element modeling.

From Equation (3), we note that the fiber effective reinforcing modulus is less than the true fiber modulus due to its curvature; there is a difference of $13 \mathrm{MPa}$ between the true and the $\mathrm{E}_{\mathrm{ERM}}$.

The effect of this difference on the homogeneous mechanical properties of steel fiber reinforced concrete will be seen in the next sub-section.

\subsection{Micromechanical Analysis}

We will illustrate how fiber curvature can be incorporated into the Mori-Tanaka micromechanical technique which approximates the interaction between the phases by assuming that each inclusion is embedded, in turn, in an infinite matrix that is remotely loaded by the average matrix strain or average matrix stress, respectively. The Mori-Tanaka method is selected due to its successful application to diverse heterogeneous material systems. It is a powerful tool for modeling multi-phase materials as well as it gives fairly good predictions of the composite stiffness (Mori and Tanaka, 1973; Benveniste, 1987). It is so important for the current work to say that the Mori-Tanaka method can model composites with random orientations of inclusions. For more derivation details, the reader is directed to the reference (Benveniste, 1987).

Here we assume that the composite is comprised of two phases; the concrete matrix with a corresponding stiffness $C_{c}$ and volume fraction $V_{c}$, while the fiber phase has a stiffness of $C_{f}$ and a volume fraction $V_{f}$. Following the standard Mori-Tanaka derivation, the expression for the dilute strain-concentration factor of the inclusion phase, $A_{f}$, can be developed. $\mathrm{A}_{\mathrm{f}}$ relates the volume-averaged strain in the inclusion to that of the matrix such that Equation (4):

$$
\left\langle\varepsilon_{\mathrm{f}}\right\rangle=\mathrm{A}_{\mathrm{f}}\left\langle\varepsilon_{\mathrm{c}}\right\rangle
$$

where Equation (5):

$$
A_{f}=\left[I+S_{f} C_{c}^{-1}\left(C_{f}-C_{c}\right)\right]^{-1}
$$

I is the fourth order identity tensor, $S_{\mathrm{f}}$ is the standard Eshelby tensor and brackets \langle\rangle denote a volume averaged quantity. Given (5), the effective composite stiffness $\mathrm{C}$ as determined using the MoriTanaka method is given as Equation (6):

$$
\mathrm{C}=\left(\mathrm{V}_{\mathrm{c}}\left\{\mathrm{C}_{\mathrm{c}}\right\}+\mathrm{V}_{\mathrm{f}}\left\{\mathrm{C}_{\mathrm{f}} \mathrm{A}_{\mathrm{f}}\right\}\left(\mathrm{V}_{\mathrm{c}} \mathrm{I}+\mathrm{V}_{\mathrm{f}}\left\{\mathrm{A}_{\mathrm{f}}\right\}\right)^{-1}\right.
$$

where, the curly brackets \{\} represent an orientational average (necessary to account for random orientations of inclusions).

Two alternative models are used to determine the effective moduli of composites containing randomly orientated steel fibers using the Mori-Tanaka method (Huang, 2001; Weng, 1990) modle. In the Huang model, the effective stiffness for a multiphase composite is found by taking the orientational average of the effective modulus for the unidirectional composite case rather than the orientational averaging of the individual components for the Weng model.

For the Weng model, the introduction of the orientational averaging of field quantities is effectively introduced before the determination of the unknown average matrix strain. For the Huang model, the unknown matrix strain is solely determined from the analysis of the unidirectional composite model and thus is not dependent on the type of randomness that the inclusions might exhibit.

Once the components of the composite effective stiffness have been determined, the standard relationships can be used to find the corresponding elastic constants. The critical step of the analysis is the determination of the dilute strain-concentration tensor $A_{f}$ relating the average strain of the inclusion to that of the matrix. In the present analysis $A_{f}$ is found via (5) where the stiffness tensor of the inclusion phase $C_{f}$ is found using the appropriate values of $\mathrm{E}_{\mathrm{ERM}}$ (see the previous section) and where the Eshelby tensors $S_{f}$ assume cylindrical inclusions (Eshelby, 1957). The steel fibers are modeled as isotropic material of modulus $\mathrm{E}_{\mathrm{ERM}}$ and a value of 0.3 for the Poisson's ratio, from which the stiffness tensor $\mathrm{C}_{\mathrm{f}}$ can be obtained in the usual manner. We thus treat the curved inclusion as a straight inclusion, but with an adjusted stiffness tensor to account for the effects of the geometry.

The mechanical properties of FRC delivered by Weng and Huang schemes are given in the Table 1.

\subsection{Interpretation}

The hook-ended fiber reinforced concrete properties obtained with the true fiber Young's modulus and $\mathrm{E}_{\text {ERM }}$ are predicted using the Weng and Huang models. The ratio of homogenous Young's modulus and Poisson's ratio obtained with effective and true fiber Young's modulus are close to 1 
which proves that the effect of hooks at the end of steel fiber on the composite can be neglected.

The role of the hooks on the overall pullout behavior was investigated by many researchers (Cunha et al., 2010). They have shown that end hook provide an increase in frictional and adhesive bond forces along the fiber which increases the pull-out resistance. It was highlighted that concrete with hookended steel fibers showed a better result than the straight fiber in the flexural and splitting tensile test because the efficiency of load transferring from matrix to fibers depends on the anchorage which is strong around hookended fibers (Eik and Puttonen, 2011). It was found also that concrete containing hook-ended steel fibers has better physical properties than that containing straight fibers.

Hence, one can conclude that the positive effect of fiber curvature in resistance and physical properties is more important than the negative neglected impact in the Young's modulus and Poisson ratio of fiber concrete composite.

The predictions given by the two micro-mechanical approaches are quite close to the experimental results (Neves and Fernandes, 2005). Hence the chosen model was found to have good accuracy in estimating homogeneous properties.

For a composite comprising $0.38 \% 3 \mathrm{D}$ random steel fibers (Table 1), one can expect an enhancement by a $200 \mathrm{MPa}$ for the Young's modulus. From Table 1, we deduce that for small volume fractions of inclusions, results from the two models are similar.

\section{MECHANICAL PROPERTIES OF CRIMPED STEEL FIBER REINFORCED CONCRETE}

It has been seen from the previous section that the effect of hook ends on the Young's modulus and Poisson's ratio can be neglected. In this section, two crimped steel fiber concrete composites are investigated (Fig. 4). In both composites the fibers are assumed to be ellipsoidal having the same mechanical properties as the true fibers.

\subsection{First Example}

Crimped steel fibers conforming to ASTM A8202001 is used in this investigation (Pawade et al., 2011b). The properties of crimped fibers are: Poisson's ratio: $\mathrm{v}_{\mathrm{f}}=$ 0.3 , Elastic modulus: $\mathrm{E}_{\mathrm{f}}=210000 \mathrm{MPa}$. Properties of silica fume concrete at 28 days of age: Poisson's ratio $\mathrm{v}_{\mathrm{c}}$ $=0.2$. Young's modulus: $\mathrm{E}_{\mathrm{c}}=31350 \mathrm{MPa}$.

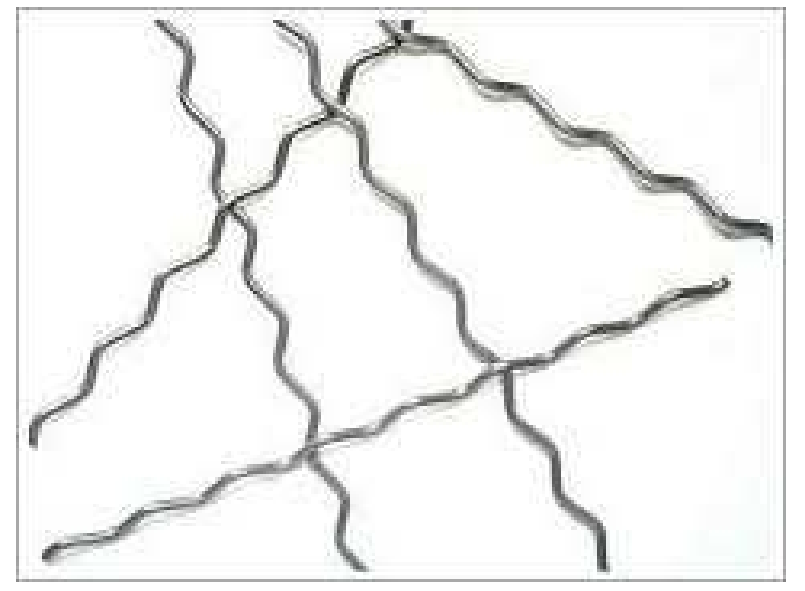

Fig. 4. Schematic representation of crimped steel fibers

Table 1. FRC with 3D randomly oriented reinforcements. A comparison between experimental data after Neves and Fernandes (2005) and micromechanical models for $0.38 \%$ steel fiber volume fraction

\begin{tabular}{lll}
\hline & Young's Modulus (MPa) & Poisson's ratio \\
\hline Experiment & 36000 & --------- \\
Weng model & 35984 & 0.2010 \\
Huang model & 36054 & 0.2004 \\
\hline
\end{tabular}

Young's modulus and Poisson's ratio of 3D randomly oriented crimped steel fiber 28 day aged concrete composite with (Length $\mathrm{L}=30 \mathrm{~mm}$, diameter D $=0.50 \mathrm{~mm}$ ) and (Length $\mathrm{L}=60 \mathrm{~mm}$, diameter $\mathrm{D}=1.00$ $\mathrm{mm}$ ) are reported in Table $\mathbf{2}$ and $\mathbf{3}$ respectively.

\subsection{Interpretation}

From Table 2, it is seen that the elastic modulus of the composite is $159 \mathrm{MPa}, 526 \mathrm{MPa}$ and $808 \mathrm{MPa}$ higher than that of concrete for steel fiber volume fraction equal to $0.5,1.0$ and $15.0 \%$ respectively.

From Table 3, it is seen that the modulus of the composite is $491 \mathrm{MPa}, 724 \mathrm{MPa}$ and $1092 \mathrm{MPa}$ higher than that of concrete for steel fiber volume fraction equal to $0.5,1.0$ and $15.0 \%$ respectively.

Our results were compared with experimental results given by Pawade et al. (2011b).

For various steel fiber volume fractions, Young's modulus predictions delivered by all models are consistent with the experiment data.

Table 2 and 3 show that the Poisson's ratio predictions delivered by all schemes are very close. 
Table 2. FRC with 3D randomly oriented crimped rein-forcements. Length, diameter, $\mathrm{L}=30$, dismeter $\mathrm{D}=0.50 \mathrm{~mm}$. A comparison between experimental data after Pawade et al. (2011b) and micromechanical models for different crimped steel fiber volume fractions

\begin{tabular}{|c|c|c|c|}
\hline Percent & & Young's modulus (MPa) & Poisson's ratio \\
\hline \multirow[t]{3}{*}{$\mathrm{V}_{\mathrm{f}}=0.5$} & Experiment & 31509 & ----------- \\
\hline & Weng model & 31590 & 0.2013 \\
\hline & Huang model & 31670 & 0.2005 \\
\hline \multirow[t]{3}{*}{$\mathrm{V}_{\mathrm{f}}=1.0$} & Experiment & 31876 & ----------- \\
\hline & Weng model & 31832 & 0.2026 \\
\hline & Huang model & 31992 & 0.2011 \\
\hline \multirow[t]{3}{*}{$V_{f}=1.5$} & Experiment & 32158 & ------------ \\
\hline & Weng model & 32074 & 0.2039 \\
\hline & Huang model & 32315 & 0.2016 \\
\hline
\end{tabular}

Table 3. FRC with 3D randomly oriented crimped reinforcements, Length, diameter, $\mathrm{mm} \mathrm{L}=60 \mathrm{~mm}=\mathrm{D}$ A comparison between experimental data after Pawade et al. (2011b) and micromechanical models for different crimped steel fiber volume fractions

\begin{tabular}{|c|c|c|c|}
\hline Percent & & Young's modulus (MPa) & Poisson'sratio \\
\hline \multirow[t]{3}{*}{$V_{f}=0.5$} & Experiment & 31841 & ----------- \\
\hline & Weng model & 31590 & 0.2013 \\
\hline & Huang model & 31670 & 0.2005 \\
\hline \multirow[t]{3}{*}{$\mathrm{V}_{\mathrm{f}}=1.0$} & Experiment & 32074 & ---------- \\
\hline & Weng model & 31832 & 0.2026 \\
\hline & Huang model & 31992 & 0.2011 \\
\hline \multirow[t]{3}{*}{$\mathrm{V}_{\mathrm{f}}=1.5$} & Experiment & 32442 & --------- \\
\hline & Weng model & 32074 & 0.2039 \\
\hline & Huang model & 32315 & 0.2016 \\
\hline
\end{tabular}

From the same tables and according to experimental results one can deduce that not only the aspect ratio can play important role in the enhancement of the composite mechanical properties but also the steel fiber length. For the same fiber volume fraction, aspect ratio and mechanical properties, when the fiber length is doubled, it is experimentally proved that the composite Young's modulus variation can reach $332 \mathrm{MPa}$. This is explained by the fact that for the same aspect ratio; $\rho$ $=\mathrm{L} / \mathrm{D}$, when the fiber length is increased, the lateral surface is increased too; $\mathrm{A}=2 \pi \mathrm{r} \mathrm{L}=\pi \mathrm{LD}=(\pi / \rho) \mathrm{L}^{2}$. Increasing the lateral surface area of a fiber increases frictional and adhesive bond forces along the fiber and leads to an increase in pull-out resistance and thus in fiber efficiency. The effect of fiber length cannot be detected by mean field homogenization models in fact only the fiber aspect ratio is taken into account in these models.

\subsection{Second Example}

Crimped steel fibers conforming to ASTM A8202001 is considered in this section (Pawade et al., 2011a) where mechanical properties are: Poisson's ratio: $\mathrm{v}_{\mathrm{f}}=$ 0.3, Elastic modulus: $E_{\mathrm{f}}=210000 \mathrm{MPa}$. Properties of silica fume concrete at 90 days of age: Poisson's ratio: $\mathrm{v}_{\mathrm{c}}$ $=0.2$. Young's modulus: $\mathrm{E}_{\mathrm{c}}=32029 \mathrm{MPa}$.

Young's modulus and Poisson's ratio of 3D randomly oriented crimped steel fiber 90 day aged concrete composite with (Length $\mathrm{L}=30 \mathrm{~mm}$, diameter D $=0.50 \mathrm{~mm}$ ) are given in Table 4 .

\subsection{Interpretation}

Our results were compared with experimental data given by Pawade et al. (2011a).

Compared to experiment data, Weng and Huang models were found to have good accuracy in estimating the homogenous Young's modulus.

From these results, it is deduced that the reinforcing effect of fibers is very important and as the crimped steel fiber content in the concrete increases, the Young's modulus increases rapidly. For $1.5 \%$ of fibers, the concrete Young's modulus is increased by a factor of 1.045 but the Poisson's ratio decreases by a factor of 1.02. From Table 4, we remark that the two schemes deliver the same prediction for the Poisson's ratio. 
Table 4. FRC with $3 \mathrm{D}$ randomly oriented crimped rein-forcements, Length, diameter, $\mathrm{L}=30 \mathrm{~mm} \mathrm{D}=0.50 \mathrm{~mm}$ A comparison between experimental data after Pawade et al. (2011a) and micromechanical models for different crimped steel fiber volume fractions

\begin{tabular}{llll}
\hline Percent & & Young's modulus (MPa) & Poisson's ratio \\
\hline $\mathrm{V}_{\mathrm{f}}=0.5$ & Experiment & 32788 & - \\
& Weng model & 32270 & 0.2013 \\
& Huang model & 32351 & 0.2005 \\
$\mathrm{~V}_{\mathrm{f}}=1.0$ & Experiment & 33159 & $-\mathbf{- - -}$ \\
& Weng model & 32511 & 0.2026 \\
$\mathrm{~V}_{\mathrm{f}}=1.5$ & Huang model & 32675 & 0.2011 \\
& Experiment & 33447 & -0.2039 \\
& Weng model & 32754 & 0.2016 \\
\hline
\end{tabular}

\section{CONCLUSION}

A model that incorporates the steel fiber curvature into micromechanical schemes via a multiphase composite approach is developed. Compared to the true Young's modulus, finite element results of embedded fibers and Voigt model show that the $\mathrm{E}_{\mathrm{ERM}}$ of the fiber is too close to its true modulus. Using the effective fiber properties, it has been shown that fiber curvature effect on the steel fiber concrete composite can be neglected.

After highlighting the influence of the steel fiber curvature, the Weng and Huang models were used to predict the elastic properties of $3 \mathrm{D}$ random orientated crimped steel fiber/concrete composites. The data delivered by the micromechanical models are compared against experiments. On examining the validity of the two models, there exists a good correlation between the predicted values and the experimental results.

\section{REFERENCES}

Benveniste, Y., 1987. A new approach to the application of Mori-Tanaka's theory in composite materials. Me-Chan. Mater., 6: 147-157. DOI: 10.1016/01676636(87)90005-6

Cunha, V.M.C.F., J.A.O. Barros and J.M.S. Cruz, 2010. Pullout behavior of steel fibers in self-compacting concrete. J. Mater. Civil Eng., 22: 1-9. DOI: 10.1061/(ASCE)MT.1943-5533.0000001

Dasari, V., P. Reddy and Y. Pawade, 2012. Combine effect of silica fume and steel fiber on mechanical properties on standard grade of concrete and their in-terrelations. Int. J. Adv. Eng. Technol., 3: 361366. DOI: $10.1098 /$ rspa.1957.0133

Eik, M. and J. Puttonen, 2011. Challenges of steel fibre reinforced concrete in load bearing structures. J. Struct. Mech., 44: 44-64.

Eshelby, J.D., 1957. The determination of the elastic field of an ellipsoidal inclusion and related problems. Proc. Roy. Soc. London, 241: 376-396. DOI: 10.1098/rspa.1957.0133
Huang, J.H., 2001. Some closed-form solutions for effective moduli of composites containing randomly orientated short fibers. Mater. Sci. Eng. A, 315: 11-20. DOI: 10.1016/S0921-5093(01)01212-6

Luca, G.S., A. Meda and G.A. Plizzari, 2006. Steel fiber concrete slabs on ground: A structural matter. ACI Struct. J., 103: 551-558.

Mori, T. and K. Tanaka, 1973. Average stress in matrix and average elastic energy of materials with misfitting inclusions. Acta Metallurgica, 21: 571-574. DOI: 10.1016/0001-6160(73)90064-3

Naaman, A.E., 2003. Engineered steel fibers with optimal properties for reinforcement of cement composites. J. Adv. Concrete Technol., 1: 241-252. DOI: 10.3151/jact.1.241

Neves, R.D. and J.C.O.F.D. Almeida, 2005. Compressive behavior of steel fibre reinforcedconcrete. Struct. Concrete, 6: 1-8. DOI: 10.1680/stco.2005.6.1.1

Oslejs, J., 2008. New Frontiers for steel fiber-reinforced concrete. Concrete Int., 30: 45-50.

Pant, A.S. and S.R. Parekar, 2009. Steel fibre reinforced concrete beams under bending, shear and tension without web reinforcement. Int. J. Recent Trends Eng., 1: 86-88.

Pawade, P.Y., A.M. Pande and P.B. Nagarnaik, 2011b. Effect of steel fibers on modulus of elasticity of concrete. Int. J. Adv. Eng. Sci. Technol., 7: 169-177.

Pawade, P.Y., P.B. Nagarnaik and A.M. Pande, 2011a. Performance of steel fiber on standard strength concrete in compression. Int. J. Civil Struct. Eng., 2: 483-492.

Ramadoss, P., V. Prabakaran and K. Nagamani, 2009. Toughness of steel fiber reinforced silica fume concrete under compression. Int. J. Applied Eng. Res., 4: 295-308.

Weng, G.J., 1990. The theoretical connection between Mori-Tanaka's. Theory and the Hashin-ShtrikmanWadpole bounds. Int. J. Eng. Sci., 28: 1111-1120. DOI: 10.1016/0020-7225(90)90111-U 